\title{
New generalized chaos-geometric and neural networks approach to nonlinear dynamics of the complex systems
}

\begin{abstract}
We present a new generalized approach to modeling nonlinear processes of chaotic systems based on the known concept of compact geometric attractors, chaos theory methods in effective realization plus implemented neural networks simulation algorithm. Using information on the phase space evolution of the nonlinear process in time and the neural networks simulation techniques can be considered as one of the fundamentally new approaches in the construction of global nonlinear prediction models for evolutionary dynamics of the complex chaotic systems and accurate description of the structure of the corresponding strange attractors.
\end{abstract}

Keywords: nonlinear dynamics, complex chaotic systems, compact geometric attractors, chaos theory methods, neural networks simulation
Volume 4 Issue 3 - 2018

\author{
Alexander $\bigvee$ Glushkov, Vasily $\bigvee$ Buyadzhi, \\ Olga Yu Khetselius, Valentin B Ternovsky \\ Department of Mathematics, Odessa State Environmental \\ University, Ukraine
}

Correspondence: AlexanderV Glushkov, Department of Mathematics, Odessa State Environmental University, Ukraine, Tel/Fax +380-482-32673, Email glushkovav@gmail.com

Received: January 27, 2018 | Published: May 10, 2018

\section{Introduction}

Multiple physical, chemical, biological, technical, communication, robotic and automation and other systems (devices) demonstrate the typical complex chaotic behaviour. In many important situations typical dynamics of these systems is the world of strong nonlinearity. In principle, the most conventional direct approach to dynamics treating problem consists in building an explanatory model using an initial data and parameterizing sources and interactions between process properties. Unfortunately, such that kind of approach is realized with difficulties and its outcomes are insufficiently correct. In the past few decades different chaos and dynamical system theories and topology models have given many useful insights to understand the output data generated by the complex nonlinear systems $\mathrm{s}^{1-12}$ especially when traditional linear models are incorrect. We are developing a new approach to modeling nonlinear processes of chaotic systems based on the known CGA concept, chaos theory methods plus implemented NNW algorithms. Using information on the phase space evolution of the nonlinear process in time and the NNW simulation techniques can be considered as one of the fundamentally new approaches in the construction of global nonlinear models of the most effective and accurate description of the structure of the corresponding attractor for studied complex system.

\section{Generalized chaos-geometric approach to complex system dynamics}

The basic idea of the construction of our approach to prediction of chaotic properties of complex systems is in the use of the traditional concept of a CGA in which evolves the measurement data, plus the NNW algorithm implementation. Let us consider some scalar measurements $s(n)=s\left(t_{0}+n \Delta t\right)=s(n)$, where $t_{0}$ is the start time, $\Delta t$ is the time step, and $n$ is the number of the measurements. The main task is to reconstruct phase space using as well as possible information contained in $s(n)$. To do it, the method of using timedelay coordinates by Packard et $\mathrm{al}^{3}{ }^{3}$ can be used, the direct using lagged variables $s(n+\tau)$ (here $\tau$ is some integer to be defined) results in a coordinate system where a structure of orbits in phase space can be captured. A set of time lags is used to create a vector in $\quad d$ dimensions, $y(n)=s(n), s(n+\tau), s(n+2 \tau), \ldots, s(n+(d-1) \tau)]$, the required coordinates are provided. Here the dimension $d$ is the embedding dimension, $d_{E}$. To determine the proper time lag at the beginning one should use the known method of the linear $A C F C_{L}(\delta)$ and look for that time lag where $C_{L}(\delta)$ first passes through $0 .{ }^{4}$ The alternative additional approach is provided by the AMI method as an approach with so called nonlinear concept of independence. The further next step is to determine the embedding dimension, $d_{E}$ and correspondingly to reconstruct a Euclidean space $R^{d}$ large enough so that the set of points $d_{A}$ can be unfolded without ambiguity. The dimension $d_{E}$ must be greater, or at least equal, than a dimension of attractor, $d_{A}$, i.e. $d_{E}>d_{A}$. To reconstruct the attractor dimension and to study the signatures of chaos in a time series, one could use such methods as the CIA by Grassberger and Procaccia ${ }^{5}$ or the FNN one by Kennel et al. ${ }^{6}$ The principal question of studying any complex chaotic system is to build the corresponding prediction model and define how predictable is a chaotic system. The new element of our approach is using the NNW algorithm in forecasting nonlinear dynamics of chaotic systems. ${ }^{9,10}$ In terms of the neuro-informatics and neural networks theory the process of modelling the evolution of the system can be generalized to describe some evolutionary dynamic neuro-equations. Imitating the further evolution of a system within NNW simulation with the corresponding elements of the self-study, self- adaptation, etc., it becomes possible to significantly improve the prediction of its evolutionary dynamics. The fundamental parameters to be computed are the Kolmogorov entropy (and correspondingly the predictability measure as it can be estimated by the Kolmogorov entropy), the LE, the KYD etc. The LE is usually defined as asymptotic average rates and they are related to the Eigen values of the linearized dynamics across the attractor. Naturally, the knowledge of the whole LE allows determining other important invariants such as the Kolmogorov entropy and the attractor's dimension. The 
Kolmogorov entropy is determined by the sum of the positive LE. The estimate of the dimension of the attractor is provided by the Kaplan and Yorke conjecture $d_{L}=j+\sum_{i=1}^{n} \lambda_{i} /\left|\lambda_{j+1}\right|$, where $j$ is such that $\sum_{i=1}^{j} \lambda_{i}>0$ and $\sum_{i=1}^{j+1} \lambda_{i}<0$ and the LE are taken in descending order. In Figure 1 we present the flowchart of the combined chaos-geometric and neural networks computational approach to nonlinear analysis and prediction of dynamics of any complex system. ${ }^{8-17}$

I. General analysis of the dynamical problem, processing dynamical variable
series for studied complex system (preliminary general analysis of
dynamics, evolutionary differential equationstreating...)

\section{ل!}

1. The Gottwald-Melboume test: $K \rightarrow 1$ - chaos;

2. Fourier decompositions, irregular nature of change-chaos;

3. Spectral analysis, Energy spectra statistics, the Wigner distribution, the spectrum of power, "Spectral nigidity";

III. The geometry of the phase space. Fractal Geometry:

4. Computation time delay $\tau$ using autocorrelation function or mutual information;

5. Determining embedding dimension $d_{E}$ by the method of correlation dimension or algorithm of false nearest neighbouning points;

6. Calculation multi-fractal spectra. Wavelet analysis;

\section{ل}

IV. Prediction model:
7. Computing global LE: $\lambda_{a} ; \mathrm{KYD} d L$, average predictability measure
$\mathrm{Pr}_{\max }$;
8. Determining the number of FNN points for the best prediction results;
9. Methods of nonlinear prediction: nonlinear parameterized function;
NNW; optimized trajectories (propagators) algonthms, ...;

Figure I Flowchart of the combined chaos-geometric approach and NNW to nonlinear analysis and prediction of chaotic dynamics of the complex systems (structures, devices).

\section{Acknowledgements}

None.

\section{Conflict of interest}

The author declares there is no conflict of interest.

\section{References}

1. Abarbanel H. Analysis of observed chaotic data. Springer; 1996.

2. Schreiber T. Interdisciplinary application of nonlinear time series methods. Phys Rep. 1999;308(1):1-64.

3. Packard N, Crutchfield J, Farmer J, et al. Geometry from a time series. Phys Rev Lett. 1980;45(9):712-716.

4. Takens F. Detecting strange attractors in turbulence. In: D Rand, L Young, editors. Dynamical Systems and Turbulence. Berlin, Springer; 1981:366338.

5. Grassberger P, Procaccia I. Measuring the strangeness of strange attractors. Phys D. 1983;9(1-2):189-208.
6. Kennel M, Brown, Abarbanel. Determining embedding dimension for phase-space reconstruction using a geometrical construction. Phys Rev A. 1992;45:3403-3411.

7. Glushkov AV. Methods of a Chaos. OSENU, Odessa. 2012.

8. Glushkov AV, Buyadzhi VV, Kvasikova AS, et al. (2017) Non-linear chaotic dynamics of quantum systems: Molecules in an electromagnetic field and laser systems. In: A Tadjer, R Pavlov, et al. editors. Quantum Systems in Physics, Chemistry, and Biology. Springer; 2017:169-180.

9. Khetselius $\mathrm{O}$ Yu. New geometric attractor and neural networks approach to studying chaotic processes in photo electronics systems. Photoelectr. 2013;22:31-37.

10. Glushkov AV, Khetselius OY, Brusentseva SV, et al. Modeling chaotic dynamics of complex systems with using chaos theory, geometric attractors, and quantum neural networks. Proc Intern Geom Center. 2014;7(3):87-94.

11. Glushkov AV, Khetselius OY, Brusentseva SV, et al. Studying interaction dynamics of chaotic systems within a non-linear prediction method: Application to neurophysiology. Advances in Neural Networks, Fuzzy Systems and Artificial Intelligence. 2014;21:69-75. 
12. Glushkov AV, Svinarenko AA, Buyadzhi VV, et al. (2014) Chaos geometric attractor and quantum neural networks approach to simulation chaotic evolutionary dynamics during perception process. Advances in Neural Networks, Fuzzy Systems and Artificial Intelligence. 2014;21:143-150.

13. Glushkov A, Khetselius O, Bunuakova Yu, et al. Sensing interaction dynamics of chaotic systems within a chaos theory and microsystem technology Geomath with application to neurophysiological systems. Sensor Electr and Microsyst Techn. 2014;11(3):62-69.

14. Glushkov AV, Ternovsky VB, Buyadzhi VV, et al. Geometry of a relativistic quantum chaos: New approach to dynamics of quantum systems in electromagnetic field and uniformity and charm of a chaos. Proc Inter Geom Center. 2014;7(4):60-71.
15. Glushkov AV, Buyadzhi VV, Ponomarenko EL. Geometry of chaos: Advanced approach to treating chaotic dynamics in some nature systems. Proc Intern Geom Center. 2014;7(1):24-29.

16. Glushkov A, Kuzakon V, Ternovsky V, Buyadzhi V (2013) Dynamics of laser systems with absorbing cell and backward-wave tubes with elements of a chaos. In: J Awrejcewicz, et al. editors. Dynamical systems theory. Lodz, WSEAS; 2013;461-466.

17. Glushkov AV, Khetselius OYu, Svinarenko AA, et al. The sea and ocean $3 \mathrm{D}$ acoustic waveguide: rays dynamics and chaos phenomena. J Acoust Soc of America. 2008;123(5): 3625. 